\title{
Centers for Medicare and Medicaid Services System for Diagnosis-Related Groups
}

National Cancer Institute

\section{Source}

National Cancer Institute. Centers for Medicare and Medicaid Services System for

Diagnosis-Related Groups. NCI Thesaurus. Code C154526.

A system developed by the Centers for Medicare and Medicaid Services to classify hospital cases into diagnosis-related groups to facilitate Medicare reimbursement. This system was used until 2007. 